\title{
White dwarfs as astrophysical probes
}

\author{
Jasonjot S. Kalirai \\ Space Telescope Science Institute, 3700 San Martin Drive, Baltimore MD, 21231 \\ email: jkalirai@stsci.edu
}

\begin{abstract}
Much of our knowledge regarding the ages of stars derives from our understanding of the Hertzsprung-Russell Diagram. The diagram is typically dominated by hydrogen burning main-sequence stars, which historically, have been used to establish our most fundamental knowledge of stellar ages and evolution. In this brief article, I highlight how deep ground and space based imaging can uncover the stellar remnants of these hydrogen burning stars, white dwarfs. We have followed up our initial discovery of several large white dwarf populations in nearby star clusters with multiobject spectrographs. The spectroscopy allows us to characterize the properties of the remnant stars (e.g., mass, temperature, and age), which are in turn used to shed new light on fundamental astrophysical problems. Specifically, we estimate the ages of the Milky Way disk and halo, provide the inputs needed to calculate the chemical evolution of galaxies, and re-iterate the important role of $\mathrm{HB}$ stars in producing the UV-upturn seen in elliptical galaxies.
\end{abstract}

Keywords. Galaxy: open clusters and associations: individual (NGC 7789, NGC 6819, and NGC 6791) - stars: evolution - techniques: photometric, spectroscopic - white dwarfs

\section{Introduction}

Ejnar Hertzsprung, in 1905, found direct evidence that stars of the same temperature, and with the same parallax (and therefore at the same distance), could have very different luminosities. His observations led him to suggest at least two broad bins that characterize stars, bright "giants" and fainter "dwarfs". The first Hertzsprung-Russell (H-R) Diagram was presented by him in 1911, consisting of a small number of nearby stars. Henry Norris Russell quickly improved the quantity and quality of data on the H-R Diagram (Russell 1913; 1914), adding more accurate observations of both field stars and nearby co-moving groups (e.g., the Hyades and the Pleiades). Russell's analysis suggested clear evidence for groupings and sequences on the H-R diagram, and even hinted at a mass-luminosity relation. For example, Russell noted that "one corner of the diagram is vacant...There do not seem to be any faint white stars".

The H-R diagram represents one of the most widely used plots in astrophysics. Much of our knowledge on the ages of stars, and our grasp of fundamental stellar evolution, is based on our ability to understand and model observables in this plane. Historically, this work has largely involved the abundant hydrogen burning stars and bright giant stars, which are both easy to see in the nearby Galaxy. With the construction of sensitive widefield imagers on 4-m and 8-m telescopes, as well as the launch of the HST, astronomers have recently been able to probe the $\mathrm{H}-\mathrm{R}$ diagram to unprecedented depths. In addition to many other discoveries, the "faint white stars" that Russell noted as being absent in the diagram, have been abundantly discovered. These stars, white dwarfs, represent the end products of low and intermediate mass hydrogen-burning stars.

White dwarfs are extremely useful probes in several avenues of stellar and galactic astrophysics. First, almost all stars will eventually form white dwarfs given the bias in the initial mass function, and therefore white dwarf represent a unique link to the distribution 

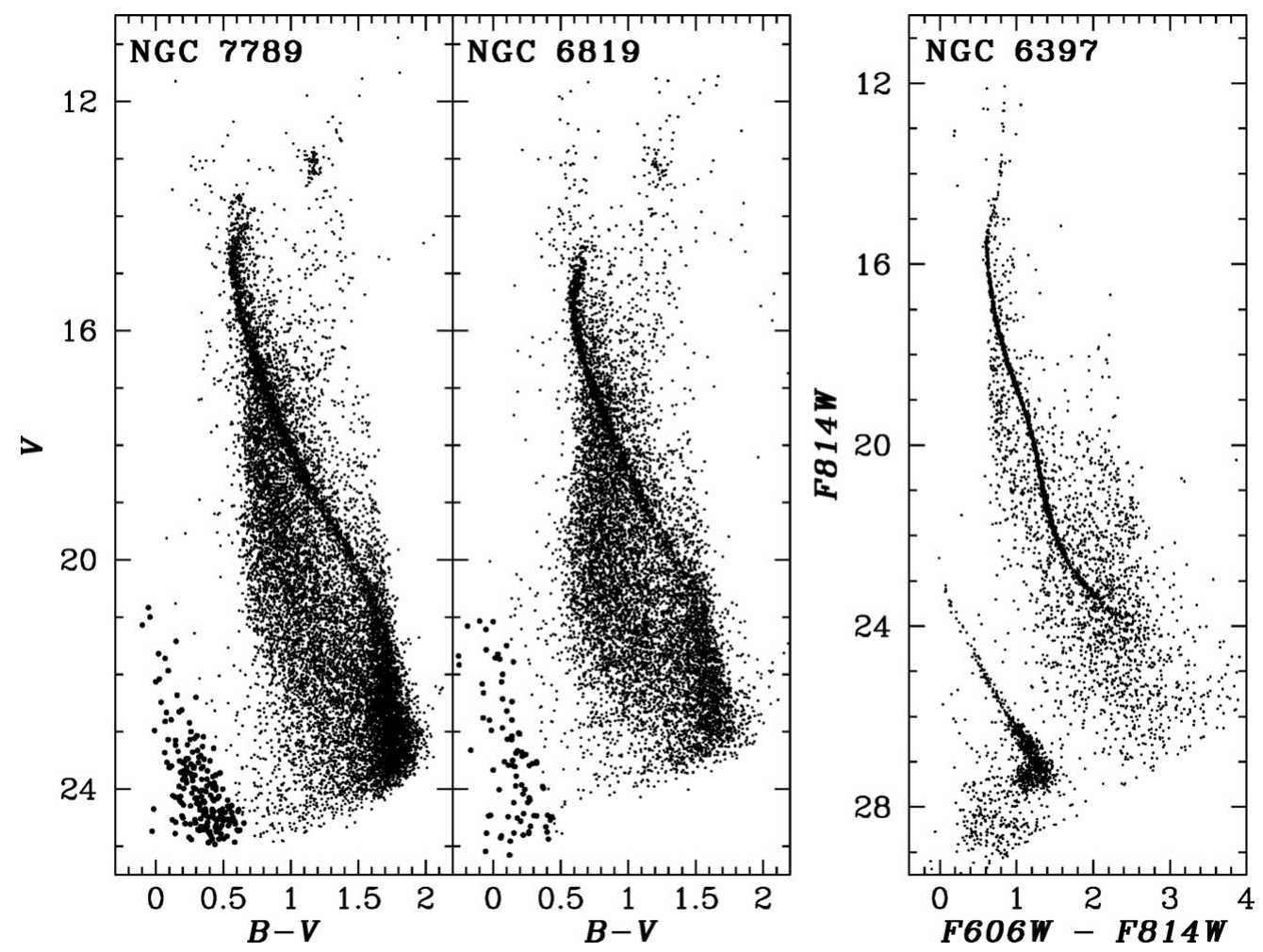

Figure 1. CFHT/CFH12K H-R diagrams of the star clusters NGC $7789(t=1.4$ Gyr $)$ and NGC $6819(t=2.5 \mathrm{Gyr})$, and an HST/ACS H-R diagram of NGC $6397(t=12 \mathrm{Gyr})$. The diagrams show both a scattered distribution of stars that are in the foreground/background of each cluster, as well as organized sequences representing the cluster stars. The main-sequences can be tracked to low mass stars (to the hydrogen burning limit in the case of NGC 6397), and the brighter giants are also detected. The faint-blue parts of the H-R diagrams show abundant populations of white dwarfs in each cluster. In the case of NGC 6397, the most massive star on the main-sequence currently has a mass of $0.8 M_{\odot}$, and therefore the white dwarfs represent the end products of stars similar to the Sun.

and properties of first generation stars in old stellar populations. Specifically, the numbers and masses of white dwarfs can be used to place constraints on the initial-mass function of a stellar population as well as an estimation of how much mass stars lose through their evolution (Reimers 1975; Renzini \& Fusi Pecci 1988; Weidemann 2000; Kalirai et al. 2008). White dwarfs also contain no nuclear energy sources and therefore simply cool and fade with time, making them excellent cosmic clocks to date stellar populations. Finally, the properties of these stars (e.g., mass and temperature) in different environments can provide detailed clues to enhance our understanding of fundamental stellar evolution.

\section{Measuring Stellar Mass Loss}

In Figure 1 we present observervational H-R diagrams of two rich open star clusters, NGC 7789 and NGC 6819, and one globular cluster, NGC 6397. The open clusters were observed with the CFH12K mosaic CCD camera on the 4-meter Canada-France-Hawaii Telescope (CFHT) as a part of the CFHT Open Star Cluster Survey (Kalirai et al. 2001a; 2001b). The globular cluster was observed as a part of a very large (123 orbit) Hubble Space Telescope (HST) allocation with the Advanced Camera for Surveys (ACS), 


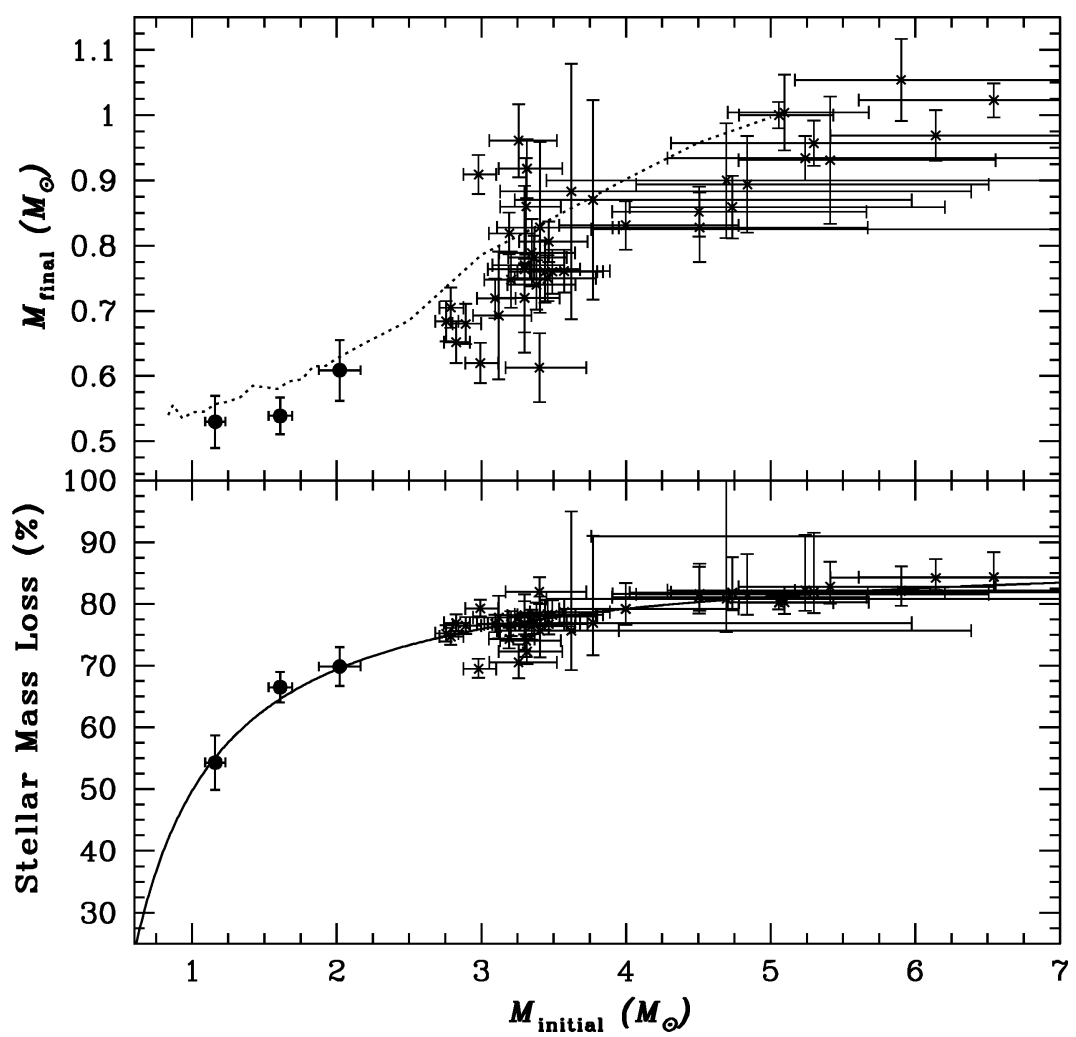

Figure 2. Top - The initial-final mass relation including all previous data (crosses) and the results from NGC 7789, NGC 6819, and NGC 6791 (larger data points). For these clusters, we have binned all white dwarfs in a given cluster and plotted a single data point indicating the weighted mean mass and $2 \sigma$ uncertainty in the mean. The dotted curve indicates the theoretical initial-final mass relation from Marigo (2001) for Solar metallicity. Bottom - The total integrated stellar mass as a function of initial mass, along with our best fit linear relation. These results suggest that the initial and final mass of Solar metallicity stars are connected by $M_{\text {final }}=(0.109 \pm 0.007) M_{\text {initial }}+0.394 \pm 0.025 M_{\odot}($ solid curve in Figure 2$)$.

Richer et al. (2006). The color-magnitude diagrams of all three clusters show rich mainsequences and stars in post-main sequence evolutionary stages. The white dwarfs that represent the end products of these stars, after they suffer mass loss along the red giant branch and asymptotic giant branch, form a tight sequence in the faint-blue part of each $\mathrm{H}-\mathrm{R}$ diagram.

We can use these white dwarfs to directly estimate how much mass normal mainsequence stars will lose through their evolution and inject back into the interstellar medium. This relation, the initial-final mass relation, is critical to our understanding of chemical evolution of galaxies, the star formation efficiency in these systems (Somerville \& Primack 1999), and is also necessary in order to use white dwarfs to date the Galactic disk and halo (e.g., Ferrario et al. 2005 and Hansen et al. 2007). We construct the relation by combining the results of the two open star clusters here with similar observations of four other clusters with different ages (e.g., Kalirai et al. 2008). A cluster with a younger age will lead to constraints on the higher mass end of the relation (since higher mass stars will evolve to white dwarfs faster), whereas an older cluster constrains the low mass end of the relation. 
The masses of the white dwarfs in each cluster are calculated by observing these stars spectroscopically (observations made with LRIS on Keck I). The spectrum of the white dwarfs contains broadened Balmer lines which are modelled (as discussed in Bergeron, Saffer, \& Liebert 1992 and Bergeron, Liebert, \& Fulbright 1995) to yield the effective temperature and gravity of each star, and therefore the mass. In the younger clusters of age $\sim 100$ Myrs, we find that the white dwarfs have masses of $\sim 1 M_{\odot}$, whereas in older 10 Gyr clusters, the stars have masses of $\sim 0.5 M_{\odot}$ (see Kalirai et al. 2005; 2007; 2008 for more information). These final remnant masses are connected with initial masses that represent the progenitor star of each white dwarf using knowledge of the cluster age and main-sequence lifetimes. For example, the difference between the cluster age and the white dwarf cooling age represents the lifetime of the progenitor star, from which we can calculate its mass. The result of this calculation, for a dozen clusters in the literature combined with our results, is illustrated in Figure 2.

The initial-final mass relation illustrates that Solar type stars will lose $\sim 50 \%$ of their mass through stellar evolution, stars with $2.5-3 M_{\odot}$ will lose $70-75 \%$ of their mass, and stars with $5-6 M_{\odot}$ will lose $\sim 80 \%$ of their mass. In Kalirai et al. (2008), we compare this relation to theoretical expectations and briefly explore the implications of the derived mass loss for chemical evolution studies. In Kalirai et al. (2007), we also find direct evidence that the masses of white dwarfs in the old, metal-rich cluster NGC 6791 are well below the expected value based on an extrapolation of the relation. Likely, this suggests that mass loss was more efficient in the higher metallicity environment. As discussed in that paper, this result naturally explains the anomalously low white dwarf cooling age measured for NGC 6791 by Bedin et al. (1995) since a large fraction of the white dwarfs have helium cores and cool slower than normal carbon-oxygen core white dwarfs.

Increased mass loss in higher metallicity environments is also likely to produce large populations of extreme horizontal branch stars, as seen in NGC 6791. The progenitors of these stars can be singly evolved red giants that lost enough mass on the red giant branch to avoid the helium flash. If the stars are well below the critical mass, they will form helium core white dwarfs. However, some of the stars that were close to the critical mass can also ignite the helium at a later stage while cooling to the white dwarf track, and subsequently populate the extreme horizontal branch (see e.g., Castellani \& Castellani 1993). This result has important implications for our understanding of the integrated colors of elliptical galaxies. These old, metal-rich systems are in some ways similar to NGC 6791, and often show UV-excesses that are easy to explain through blue horizontal branch morphologies.

\section{The Age of the Galactic Disk and Halo}

The initial-final mass relation derived above represents an important ingredient to properly use white dwarfs as chronomoters to date stellar populations, such as the Milky Way disk and halo. As illustrated in the right panel of Figure 1, deep HST observations can uncover even the detailed white dwarf cooling sequences in the nearest globular clusters, such as M4 (Richer et al. 2004; Hansen et al. 2004), NGC 6397 (Richer et al. 2006; Hansen et al. 2007), and 47 Tuc (pending cycle 17 HST/ACS program). Modelling the luminosity and color functions of the white dwarfs in these halo clusters, through simulations that propogate main-sequence stars with a given mass function through the initial-final mass relation to the white dwarf stage, suggest an age of $\sim 12$ Gyr for the clusters (see e.g., Hansen et al. 2007). For the field white dwarf population of the Milky Way disk, a similar analysis suggests a significantly younger age of $\sim 8$ Gyr (see Figure 3). 

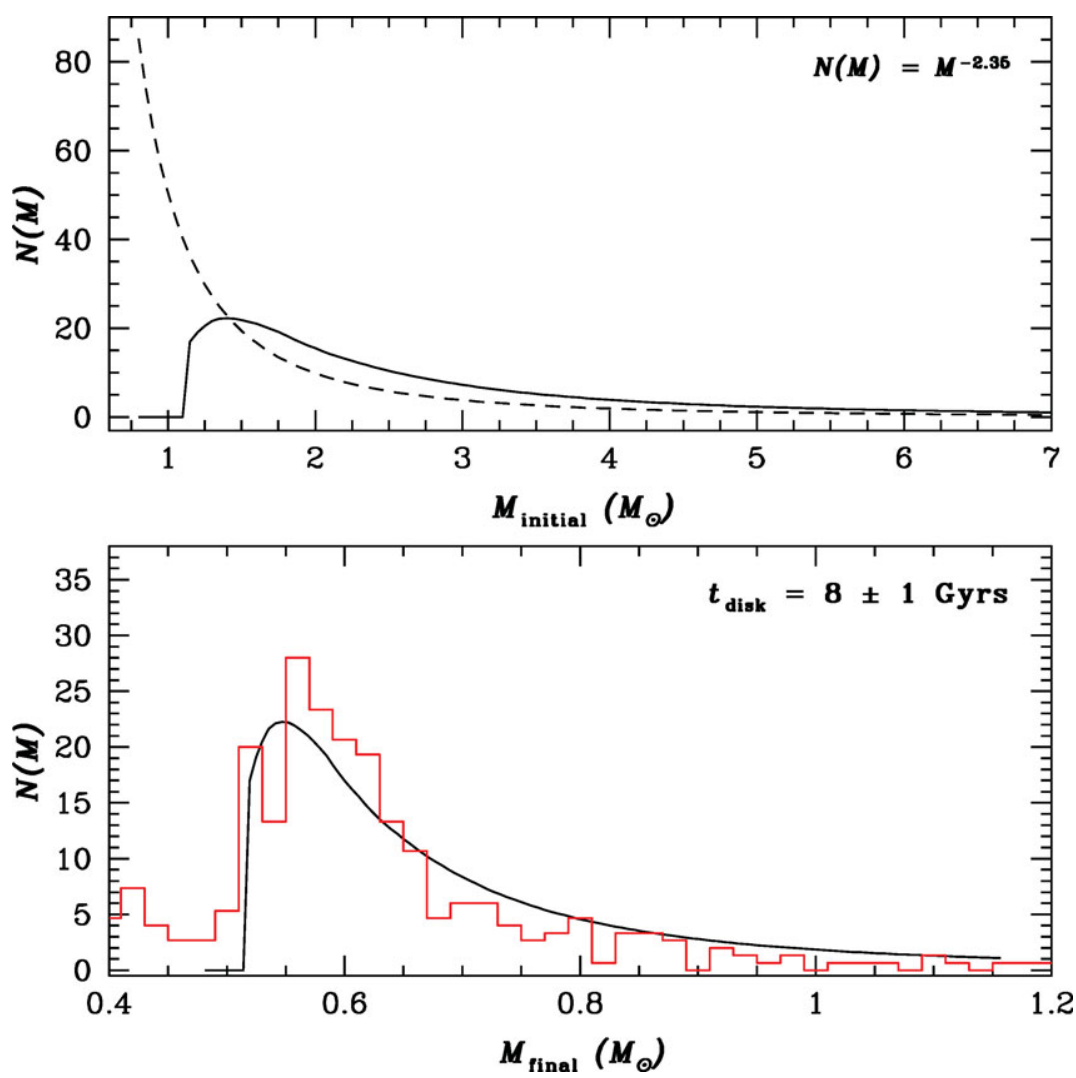

Figure 3. A Salpeter mass function (dashed curve) illustrates the large number of low mass stars that will be formed over any stellar population's lifetime (top panel). The solid curve shows a cutoff imposed by the star formation history of the population (i.e., we assume that the population has formed stars continously over the past 8 Gyr, and only those stars that would have evolved to white dwarfs in that time are shown). Propogating this function through the initial-final mass relation yields a nice fit to the observed field white dwarf luminosity function of the Milky Way disk (bottom panel).

\section{Conclusions}

White dwarfs are extremely interesting stellar probes to improve our understanding of several astrophysical problems. Although difficult to observe given their faintness, high quality imaging and spectroscopic observations of these stars can be obtained with both ground and space based telescopes. These data have provided new constraints on our understanding of stellar mass loss and evolution, and have been used to provide independent age estimates for the Galactic disk and halo. The properties of white dwarfs in different environments can also improve our general understanding of chemical evolution in galaxies and the stellar content of elliptical galaxies.

\section{References}

Bedin, L. R., Salaris, M., Piotto, G., King, I. R., Anderson, J., Cassisi, S., \& Momany, Y. 2005, ApJL, 624, 45

Bergeron, P., Saffer, R. A., \& Liebert, J. 1992, ApJ, 394, 228

Bergeron, P., Liebert, J., \& Fulbright, M. S. 1995, AJ, 444, 810

Castellani, M. \& Castellani, V. 1993, ApJ, 407, 649

Ferrario, L., Wickramasinghe, D., Liebert, J., \& Williams, K. A. 2005, MNRAS, 361, 1131 
Hansen, B. M. S., et al. 2004, ApJS, 155, 551

Hansen, B. M. S., et al. 2007, ApJ, 671, 380

Hertzsprung, E. 1905, Zeitschrift fur Wissenschaftliche Photographie, 3, 442

Hertzsprung, E. 1911, Publ. Astrophys. Observ. Potsdam, 22, 1

Kalirai, J. S., Richer, H. B., Fahlman, G. G., Cuillandre, J., Ventura, P., D’Antona, F., Bertin, E., Marconi, G., \& Durrell, P. 2001a, AJ, 122, 257

Kalirai, J. S., Richer, H. B., Fahlman, G. G., Cuillandre, J., Ventura, P., D’Antona, F., Bertin, E., Marconi, G., \& Durrell, P. 2001b, AJ, 122, 266

Kalirai, J. S., Richer, H. B., Reitzel, D., Hansen, B. M. S., Rich, R. M., Fahlman, G. G., Gibson, B. K., \& von Hippel, T. 2005, ApJL, 618, L123

Kalirai, J. S., Bergeron, P., Hansen, B. M. S., Kelson, D. D., Reitzel, D. B., Rich, R. M., \& Richer, H. B. 2007, ApJ, 671, 748

Kalirai, J. S., Hansen, B. M. S., Kelson, D. D., Reitzel, D. B., Rich, R. M., \& Richer, H. B. 2008, ApJ, 676, 594

Marigo, P. 2001, A\&A A, 370, 194

Reimers, D. 1975, Societe Royale des Sciences de Liege, Memoires, 8, 369

Renzini, A. \& Fusi Pecci, F. 1988, ARAA, 26, 199

Richer, H. B. et al. 2004, AJ, 127, 2771

Richer, H. B. et al. 2006, Science, 313, 936

Russell, H. N. 1913, The Observatory, 36, 324

Russell, H. N. 1913, The Observatory, 37, 165

Somerville, R. S. \& Primack, J. R. 1999, MNRAS, 310, 1087

Weidemann, V. 2000, A\& A, 363, 647

\section{Discussion}

M. Pinsonneault: Can you comment on the potential implications of the high He WD fraction on the age of NGC 6791 ?

J. KALIRAI: This can likely explain the anomalously- low WD cooling age of NGC 6791. He-core WDs cool a factor of 3 times slower than C-O core WDs, and therefore there is an expectation for a brighter WD peak in the cluster luminosity function, as detailed by Bedin et al. The result also naturally explains the large number of EHB stars in this cluster.

S. YI: If $M_{\mathrm{WD}} \sim 0.5$, as you find, we expect to see a lot more PAGB stars than found in GCs, because low- $M$ PAGB stars live long. This was also a problem in Brown et al.'s M32 observation. Any comment?

J. KALIRAI: Changing the mass could be one of several explanations for this. The nuclear timescales may not be the dominant mechanism, and the TP-AGB models are very uncertain. The result is consistent with Brown et al.'s M32 observations given that the lifetimes may not scale strongly with mass.

K. Williams: A comment that the initial-final mass relation, especially at the high-mass end, is very sensitive to the input cluster ages (see Salaris et al. 2008). We need to be cautious to avoid circular arguments in using WDs to get ages in younger open clusters. 
J. KALIRAI: I fully agree. This is especially true at young ages where the MS turnoff morphology is vertical in an optical CMD. Alternate age measurements, such as lithium or MS turn- on ages will be required.

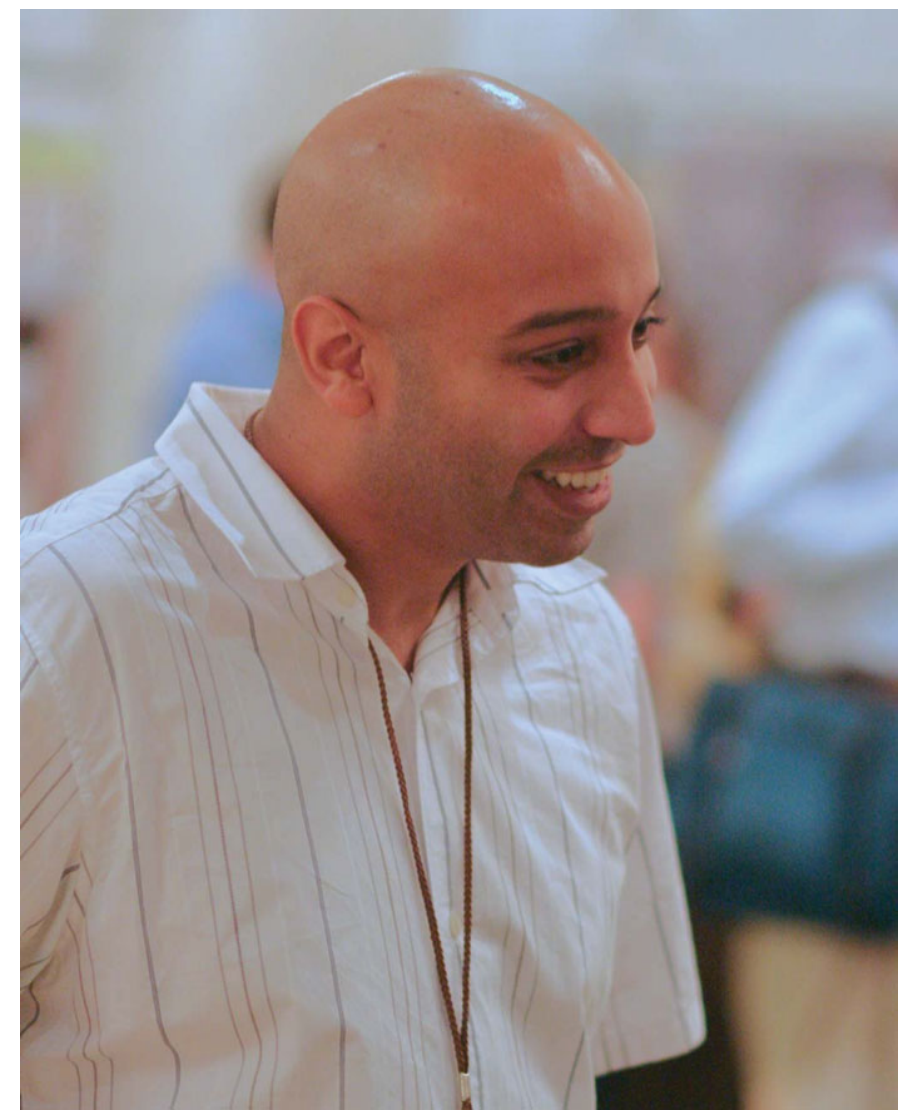

Jasonjot Kalirai 


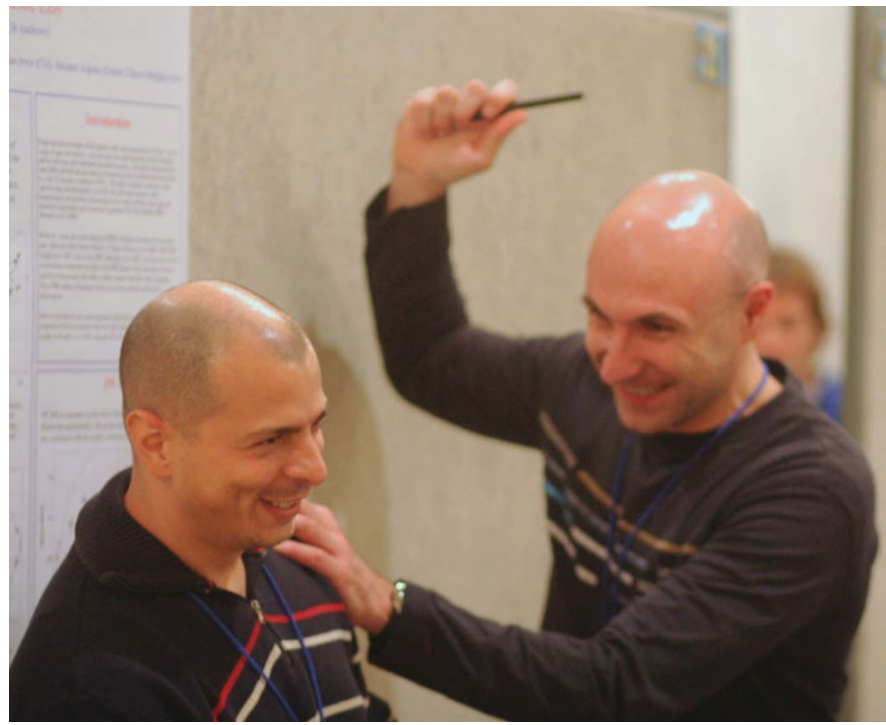

Andrea Bellini and Maurizio Salaris

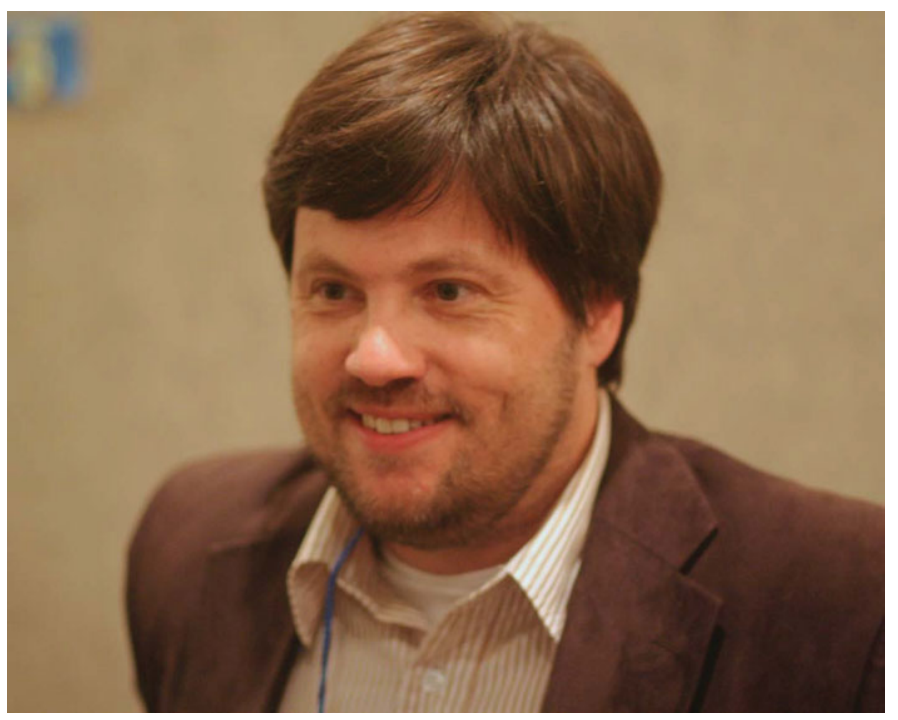

Andreas Kaufer 